\title{
On the Prüfer rank of mutually permutable products of abelian groups
}

\author{
A. Ballester-Bolinches ${ }^{1} \cdot$ John $^{\text {Cossey }}{ }^{2} \cdot$ H. Meng ${ }^{1} \cdot$ M. C. Pedraza-Aguilera ${ }^{3}$ \\ Received: 19 July 2018 / Accepted: 6 October 2018 / Published online: 16 October 2018 \\ (c) Fondazione Annali di Matematica Pura ed Applicata and Springer-Verlag GmbH Germany, part of Springer \\ Nature 2018
}

\begin{abstract}
A group $G$ has finite (or Prüfer or special) rank if every finitely generated subgroup of $G$ can be generated by $r$ elements and $r$ is the least integer with this property. The aim of this paper is to prove the following result: assume that $G=A B$ is a group which is the mutually permutable product of the abelian subgroups $A$ and $B$ of Prüfer ranks $r$ and $s$, respectively. If $G$ is locally finite, then the Prüfer rank of $G$ is at most $r+s+3$. If $G$ is an arbitrary group, then the Prüfer rank of $G$ is at most $r+s+4$.
\end{abstract}

Keywords Abelian group · Soluble group · Polycyclic group · Rank · Factorisations

Mathematics Subject Classification 20D10 - 20D20

\section{Introduction}

A group $G$ has finite (or Prüfer or special) rank $r=r(G)$ if every finitely generated subgroup of $G$ can be generated by $r$ elements and $r$ is the least integer with this property. Denote also by $d(G)$ the minimum number of elements required to generate the group $G$. If the locally soluble group $G=A B$ is the product of two subgroups $A$ and $B$ with finite Prüfer rank, then

$凶 \quad$ A. Ballester-Bolinches

Adolfo.Ballester@uv.es

John Cossey

john.cossey@anu.edu.au

H. Meng

hangyangmenges@gmail.com

M. C. Pedraza-Aguilera

mpedraza@mat.upv.es

1 Departament de Matemàtiques, Universitat de València, Dr. Moliner 50, 46100 Burjassot, València, Spain

2 Department of Mathematics, Mathematical Sciences Institute, Australian National University, Canberra, ACT 2601, Australia

3 Instituto Universitario de Matemática Pura y Aplicada, Universitat Politècnica de València, Camino de Vera, 46022 Valencia, Spain 
$G$ is hyperabelian with finite Prüfer rank (see [3, Theorem 1.1]) and, in this case, the Prüfer rank of $G$ is bounded by a function of the Prüfer ranks of $A$ and $B$ (see [1, Theorem 4.3.5]). Unfortunately, this bound is not explicit. If $G$ is a finite $p$-group for some prime $p$ and the Prüfer ranks of $A$ and $B$ are bounded by $r$, then the Prüfer rank of $G$ is bounded by a polynomial function of $r$. Better bounds for factorised finite $p$-groups were shown in [2]. However, it seems to be difficult to decide if the Prüfer rank of $G$ is bounded by a linear function of the Prüfer ranks of $A$ and $B$. On the other hand, the class of metabelian groups of finite rank which are products of two abelian subgroups has attracted growing interest recently, particulary in relation to the class of all metabelian groups which are constructible in the sense of Baumslag and Bieri [5].

Our main goal in this paper is to give a linear explicit bound for the Prüfer rank of a mutually permutable product $G=A B$ of two abelian subgroups $A$ and $B$ in terms of the Prüfer ranks of $A$ and $B$.

Our first theorem gives an upper bound for the Prüfer rank of locally finite mutually permutable products.

Theorem A Let the locally finite group $G=A B$ be the mutually permutable product of the abelian subgroups $A$ and $B$. If $A$ and $B$ have Prüfer ranks $r$ and $s$, respectively, then the Prüfer rank of $G$ is at most $r+s+3$.

As a consequence, a linear upper bound for the Prüfer rank of an arbitrary mutually permutable product is obtained.

Theorem B Let the group $G=A B$ be the mutually permutable product of the abelian subgroups $A$ and $B$. If $A$ and $B$ have Prüfer ranks $r$ and $s$, respectively, then the Prüfer rank of $G$ is at most $r+s+4$.

Recall that two subgroups $A$ and $B$ of a group $G$ permute if $A B=B A$ is a subgroup of $G$. $A$ and $B$ are called mutually permutable if every subgroup of $A$ permutes with $B$ and every subgroup of $B$ permutes with $A$; of course any two normal subgroups are mutually permutable. If every subgroup of $A$ permutes with every subgroup of $B$, we say that $A$ and $B$ are totally permutable. We say that a group $G$ is the mutually (respectively, totally) permutable product of the subgroups $A$ and $B$ if $G=A B$ and $A$ and $B$ are mutually (respectively, totally) subgroups of $G$. Obviously totally permutable subgroups are mutually permutable but the converse does not hold in general. The structure of mutually and totally permutable products has been investigated by several authors in the last 25 years, especially in the finite case, and received a full discussion in [4]. Mutually permutable products of infinite groups were considered in $[6,7]$. They play an important role in the proof of our main theorems.

Throughout the paper, the word rank will mean Prüfer rank.

\section{Preliminary results}

We collect in this section some results which are needed in the proof of main theorems. The following known property about ranks can be found in [9, Lemma 1.6.23] and will be used in the sequel without further comment: Let $N \unlhd G$ and $H \leq G$. Then $r(G) \leq r(G / N)+r(N)$ and $r(H) \leq r(G)$.

We need to use power automorphisms of a group, so perhaps a quick review of facts about these groups would be appropriate. Recall that the power automorphism group of a group $G$, PAut $(G)$, is the set of all automorphisms of $G$ which leave every subgroup of $G$ 
invariant. Hence, if $\alpha \in \operatorname{PAut}(G)$, there exists an integer $n_{g, \alpha}$ such that $g^{\alpha}=g^{n_{g, \alpha}}$, for all $g \in G$. If $n_{g, \alpha}=n_{\alpha}$ does not depend on the choice of $g$, then $\alpha$ is called a universal power automorphism.

The structures of $G$ and $\operatorname{PAut}(G)$ are strictly linked. For instance, if $G$ is a finite abelian $p$-group or more generally a finite regular $p$-group [8, Theorem 5.3.1], then every power automorphism is universal, and via the restriction homomorphism $\operatorname{PAut}(G)$ can be embedded in $\operatorname{Aut}(\langle g\rangle)$, for every cyclic group $\langle g\rangle$ of $G$ of maximal order. In fact, we have:

Lemma 1 [8, Theorem 3.4.1] Every power endomorphism of an abelian group $G$ is locally universal.

In particular, if $G$ is finite and abelian of exponent $p^{n}$, then $\operatorname{PAut}(G)$ can be embedded in $\operatorname{Aut}\left(C_{p^{n}}\right)$.

Another well-known fact we shall need is the description of the automorphism group of a cyclic group. We state it here for the sake of completeness.

Lemma 2 [10, I, Satz 13.19] Let $G$ be a cyclic group of order $p^{n}$, $p$ a prime.

(i) If $p$ is odd, then $\operatorname{Aut}(G) \simeq C_{p^{n-1}(p-1)}$.

(ii) If $p=2$, then

(a) if $n=1$, then $\operatorname{Aut}(G)=1$;

(b) if $n=2$, then $\operatorname{Aut}(G)=C_{2}$;

(c) if $n>2$, then $\operatorname{Aut}(G)=C_{2} \times C_{2^{n-2}}$.

Moreover, consider a cyclic group $C=\langle c\rangle$ of order $2^{n}, n>2$. Then $\operatorname{Aut}(C)=\langle u\rangle \times\langle\alpha\rangle$, where $c^{u}=c^{-1}$ and $c^{\alpha}=c^{5}$ and the order of $\alpha$ is $2^{n-2}$. In particular, the involutions of $\operatorname{Aut}(C)$ are exactly $u, \gamma=\alpha^{2^{n-3}}$ and $\eta=u \gamma$. Furthermore, $c^{\gamma}=c^{2^{n-1}+1}$ and $c^{\eta}=c^{2^{n-1}-1}$.

Assume that a group $A$ acts on a group $B$. Let $a \in A$ and $b \in B$. We say that $a$ inverts $b$ if $b^{a}=b^{-1} ; a$ inverts $B$ if $a$ inverts every element of $B$.

The following lemma shows that, in totally permutable products of finite $p$-groups, the structure of a core-free factor is very restricted.

Lemma 3 Let the group $G=A B$ be the totally permutable product of finite abelian p-groups $A$ and $B$. Assume that $\operatorname{Core}_{G}(A)=1$.

(i) If $p$ is odd, then A is cyclic.

(ii) If $p=2$, then either $A$ is cyclic or $A=\langle a\rangle \times\langle c\rangle$ for elements $a, c \in A$, such that $o(a)=2$ and $a$ inverts $B$.

Proof First of all note that $A \cap B$ is a normal subgroup of $G$ contained in $A$. Hence, $A \cap B \leq$ Core $_{G}(A)=1$.

Let $a \in A$ be an element of order $p$ and let $X$ be a cyclic subgroup of $B$. Then $X$ is a maximal subgroup of $X\langle a\rangle$ and so $a$ normalises $X$. Therefore, $a$ acts as a power automorphism on $B$. By Lemma 1, $a$ acts as a universal power automorphism on $B$. Since $\operatorname{Core}_{G}(A)=1$, it follows that $a$ acts nontrivially on $B$. Hence, $\Omega_{1}(A)$ can be embedded in $\operatorname{PAut}(B)$. By Lemma $1, \Omega_{1}(A)$ is isomorphic to a subgroup of $\operatorname{Aut}\left(C_{p^{n}}\right)$, where $\exp (B)=p^{n}$.

If $p$ is odd, PAut $(B)$ is cyclic by Lemma 2(i) and so is $\Omega_{1}(A)$. Then, being $A$ the direct product of cyclic subgroups, $A$ is cyclic. This establishes (i).

Assume that $p=2$ and $A$ is not cyclic. Then, by Lemma 2(ii), $\Omega_{1}(A)$ is a subgroup of the direct product of a cyclic 2-group and a group of $\operatorname{order} 2, \exp (B) \geq 8$, and there 
exists an element $a \in A$ that inverts $B$ by conjugation. Suppose that there exists an element $x \in A$ with $x^{2}=a$. Applying [6, Lemma 6], we have that $\left[x^{2}, y^{2}\right]=1$ for all $y \in B$. Since conjugation by $a$ inverts $y$, we must have $y^{4}=1$ for all $y \in B$, so that we are forced to the contradiction $\exp (B)=4$. Hence, $a \notin \Phi(A)$ and $\langle a\rangle$ has a complement in $A$. By Lemma 2(ii), $A=\langle a\rangle \times\langle c\rangle$ for some $c \in A$. This establishes (ii).

According to [8], a group is called weak if it is generated by its elements of infinite order. In particular, a nilpotent group is weak if it contains an element of infinite order.

Lemma 4 Let the group $G=A B$ be the totally permutable product of the abelian subgroups $A$ and $B$. Assume that $\operatorname{Core}_{G}(A)=1$ and $B$ is weak. Then $B$ is normal in $G$ and $|G: B| \leq 2$.

Proof We may assume that $B$ is a proper subgroup of $G$. Note that $A \cap B=1$. Let $x \in B$ be an element of infinite order and $a \in A$. If $a$ is of infinite order, then $\left\langle a^{2}\right\rangle$ is normalised by $x$ by [7, Lemma 1(2)] and if $a$ has finite order, $\left\langle a^{2}\right\rangle$ is normalised by $x$ by [7, Lemma 3]. Since $B$ is weak, it is generated by elements of infinite order. Therefore, $\left\langle a^{2}\right\rangle$ is normal in $G$. Since $\operatorname{Core}_{G}(A)=1$, it follows that $a^{2}=1$.

Therefore, it has been proved that $A$ is an elementary abelian 2-group. Consider $a \in A$ and $x \in B$. Then $|\langle a\rangle\langle x\rangle:\langle x\rangle|=2$. Hence, $\langle x\rangle$ is normalised by $A$. Hence, $A$ can be embedded in PAut $(B)$ since $\mathrm{C}_{A}(B) \leq \operatorname{Core}_{G}(A)=1$. Applying [8, Corollary 4.2.3], $A$ has at most order 2. Thus, $B$ has index at most 2 in $G$ and so it is normal in $G$, as required.

Our next lemma is a result of Lucchini concerning the number of generators of a finite group. It will be essential in the proof of Theorem A.

Lemma 5 [13, Lemma 1] Let $G$ be a finite group with a normal p-subgroup N. Assume that the Sylow p-subgroups of $G$ can be generated by $r$ elements.

(a) If $G / N$ can be generated by delements, where $d \geq r+1$, then $G$ can be generated by $d$ elements.

(b) If $p=2$ and $G / N$ can be generated by $d$ elements, where $d \geq r$, then $G$ can be generated by d elements.

The following result is a particular case of a more general result of Lucchini (see [14, Theorem 3]). We include a proof for the sake of completeness.

Lemma 6 Let $G$ be a finite soluble group. Suppose that the Sylow 2-subgroups of $G$ can be generated by $d+1$ elements and the other Sylow subgroups of $G$ can be generated by $d$ elements. Then $G$ can be generated by $d+1$ elements.

Proof We argue by induction on $|G|$. Since $G$ is soluble, there exists a nontrivial normal $p$-subgroup $N$ of $G$ for some prime $p$. The hypotheses of the lemma hold in $G / N$. Hence, $G / N$ can be generated by $d+1$ elements by induction. Let $P$ be a Sylow $p$-subgroup of $G$. If $p=2$, then $P$ can be generated by $d+1$ elements. Applying Lemma $5(b)$, we have that $G$ can be generated by $d+1$ elements. If $p \neq 2$, then $P$ can be generated by $d$ elements. It follows from Lemma 5 ( $a$ ) that $G$ can be generated by $d+1$ elements.

Recall that $d(G)$ is the minimum number of elements required to generate the group $G$. Now define $f(G)$ to be the maximum of $\{d(G / H)\}$ for every normal subgroup $H$ of finite index in $G$. From [12, Theorem], we have the following:

Lemma 7 If $G$ is a polycyclic group, then $d(G) \leq f(G)+1$. 


\section{Ranks of finite $p$-groups}

Some results about the rank of a finite $p$-group, $p$ a prime, which is a mutually permutable product of two abelian subgroups will be proved in this section. These results will be crucial to prove our main theorems.

Note that if $A$ is a finite abelian $p$-group, then $d(A)=r(A)$.

Lemma 8 Let the finite p-group $G=A B$ be the product of two cyclic subgroups $A$ and $B$. Then the rank of $G$ is at most 2 if $p$ is odd and at most 3 if $p=2$.

Proof If $p>2$, then $G$ is metacyclic by [10, III, Satz 11.5]. Thus, $G$ is of rank 2. Assume that $p=2$. If $G$ is a nonmetacyclic 2-group, then $G$ has a unique nonmetacyclic maximal subgroup by [11, Theorem 5.1]. Let $M$ be a metacyclic maximal subgroup of $G$. Then $M$ is normal in $G$ and $G / M$ is cyclic. Hence, $r(G) \leq r(M)+r(G / M) \leq 2+1=3$, as required.

Lemma 9 Let $G=\langle a\rangle\langle b\rangle$ be the product of two cyclic groups $\langle a\rangle$ and $\langle b\rangle$ such that $|\langle a\rangle|=$ $|\langle b\rangle|=2^{2}$ and $\langle a\rangle \cap\langle b\rangle=1$. Then $\left\langle a^{2}, b^{2}\right\rangle$ is contained in $\mathrm{Z}(G)$. Furthermore, if $G$ is nonmetacyclic, then $[a, b]=a^{2} b^{2}$.

Proof By [4, Corollary 3.1.9], $G$ is the totally permutable product of the subgroups $\langle a\rangle$ and $\langle b\rangle$. Then $\left\langle a^{2}\right\rangle\langle b\rangle$ is a subgroup of $G$ and $\langle b\rangle$ is a normal subgroup of $\left\langle a^{2}\right\rangle\langle b\rangle$. If $a^{2}$ does not centralise $\langle b\rangle$, we have that $a^{2}$ inverts $\langle b\rangle$. In this case $\langle b\rangle\left\langle a^{2}\right\rangle$ is isomorphic to the dihedral group of order 8. Hence, $\langle b\rangle$ is a characteristic subgroup of $\langle b\rangle\left\langle a^{2}\right\rangle$ which is normal in $G$. Hence, $\langle b\rangle \unlhd G$ and then $b^{a} \in\langle b\rangle$. It follows that $b^{a}=b$ or $b^{a}=b^{-1}$. In both cases, we have $b^{a^{2}}=b$, against supposition. Hence, $\left[a^{2}, b\right]=1$ and $a^{2} \in \mathrm{Z}(G)$. By using the same arguments with $b^{2}$, we get $\left\langle a^{2}, b^{2}\right\rangle \leq \mathrm{Z}(G)$. Assume now $G$ is a nonmetacyclic group. By [11, Proposition 2.12], $\Phi(G)=\left\langle a^{2}, b^{2}\right\rangle$ and $G /\left\langle a^{2}, b^{2}\right\rangle$ is abelian. Hence, $[a, b] \in\left\langle a^{2}\right\rangle\left\langle b^{2}\right\rangle$. Since $[a, b] \neq 1$, we deduce that $[a, b]=a^{2}, b^{2}$ or $a^{2} b^{2}$. If $[a, b]=a^{2}$ or $[a, b]=b^{2}$, then either $\langle a\rangle$ or $\langle b\rangle$ is a normal subgroup of $G$ and $G$ is metacyclic. By this contradiction $[a, b]=a^{2} b^{2}$, as required.

Lemma 10 Let $G=A B$ be the mutually permutable product of the abelian 2-groups $A$ and $B$ with $A \cap B=1$. Assume that $s=r(B) \leq 2$. If $A$ is either cyclic or $A=\langle a\rangle \times\langle y\rangle$ such that $o(y)=2$ and $y$ inverts $B$, then $r(G)$ is at most $s+3$.

Proof Since $A \cap B=1$, we have that $A$ and $B$ are totally permutable subgroups of $G$ by [4, Proposition 4.1.16]. Let $D=\operatorname{Core}_{G}(B)$. By [4, Lemma 4.1.10], $G / D$ is the totally permutable product of the subgroups $A D / D$ and $B / D$. Moreover, $B / D$ is core-free in $G / D$. By Lemma 3, either $B / D$ is cyclic or $B / D=\langle b D\rangle \times\langle x D\rangle$, where $o(b D)=2^{m}$ and $o(x D)=2$. Note that if $g \in D$, then $g^{z} \in\langle g\rangle\langle z\rangle \cap D=\langle g\rangle(\langle z\rangle \cap D)=\langle g\rangle$ for all $z \in A$. Therefore, $\langle g\rangle$ is a normal subgroup of $G$.

We distinguish two cases:

(i) $r(B)=1$. Let $C$ be a cyclic subgroup of $A$ such that $|A: C| \leq 2$. Then $C B$ is a normal subgroup of $G$ such that $r(C B) \leq 3$ by Lemma 8 , and $G / C B$ is cyclic. Therefore, $r(G) \leq 4$ and the lemma holds in this case.

(ii) Assume that $r(B)=2$. If $B / D$ is cyclic, then $D$ is not contained in $\Phi(B)$, the Frattini subgroup of $B$. Let $u \in D \backslash \Phi(B)$. Then $U=\langle u\rangle$ is a normal subgroup of $G$. Since $B / \Phi(B)$ is an elementary abelian group of order 4 and $U \Phi(B)$ is a proper subgroup of $B$, it follows that $B / U \Phi(B)$ is cyclic of order 2 . In addition $U \Phi(B) / U$ is contained in $\Phi(B / U)$, so that 
$B / U$ is cyclic. Since $G / U$ is the totally permutable product of the subgroups $A U / U$ and $B / U$ by $[4$, Lemma 4.1.10], $G / U$ satisfies the hypotheses of the theorem. Since $r(B / U)=1$, we have that $r(G / U) \leq 4$ by Case (i). Therefore, $r(G) \leq 5$.

Suppose that $B / D$ is not cyclic. Then $B / D=\langle b D\rangle \times\langle x D\rangle$, where $o(b D)=2^{m}$ and $o(x D)=2$. In this case, $x^{2} \in D$ and so $\left\langle x^{2}\right\rangle$ is a normal subgroup of $G$. The lemma will therefore follow should we succeed in proving that $r\left(G /\left\langle x^{2}\right\rangle\right) \leq 4$. Without loss of generality, we may assume that $x^{2}=1$. Since $x D \notin \Phi(B / D)$, we have that $x$ is an element of order 2 in $B \backslash \Phi(B)$. Let $M$ be a complement of $\langle x\rangle$ in $B$. Now $B$ cannot be cyclic. Hence, $M=\langle b\rangle$ is cyclic for some $b \in B$, and $B=\langle b\rangle \times\langle x\rangle$.

Assume that $A$ is cyclic. Then $r(A\langle b\rangle) \leq 3$ by Lemma 8 , and $A\langle b\rangle$ is a normal subgroup of $G$ such that $G / A\langle b\rangle$ is cyclic. Therefore, $r(G) \leq 4$.

Assume that $A=\langle a\rangle \times\langle y\rangle$ such that $o(y)=2$ and $y$ inverts $B$. Write $N=\langle a\rangle\langle b\rangle$. Then $N$ is a normal subgroup of $G$ and $G / N \simeq C_{2} \times C_{2}$. By Lemma 8 , we have that $r(N) \leq 3$ and then that $r(G) \leq 5$. What we must prove is that $d(H) \leq 4$ for all subgroups $H$ of $G$. Assuming this to be false, let us choose a subgroup $H$ of $G$ such that $d(H) \geq 5$.

Since $d(H) \leq r(G) \leq 5$, we have that $d(H)=5$. If $H N / N$ is cyclic, we have $d(H) \leq$ $r(H) \leq r(H / H \cap N)+r(N) \leq 1+3=4$, a contradiction which shows that $G=H N$. Denote $H_{1}=H \cap N$. Then we have $H / H_{1} \simeq G / N \simeq C_{2} \times C_{2}$ and so $d\left(H_{1}\right) \geq 3$. Since $d\left(H_{1}\right) \leq r(N) \leq 3$, we have that $d\left(H_{1}\right)=3$ and so $H_{1}$ is not metacyclic. Note that $H_{1}$ cannot be equal to $N$ because $d(N)=2$. Therefore, $H_{1}$ lies inside a nonmetacyclic maximal subgroup $X$ of $N$. Applying [11, Theorem 5.1], it follows that $X=\left\langle a b, a^{2}, b^{2}\right\rangle$, $d(X)=3$, and $o(a), o(b) \geq 4$. By [11, Proposition 2.12], we have that $\Phi(\Phi(N))=\left\langle a^{4}, b^{4}\right\rangle$ and $|N: \Phi(\Phi(N))|=2^{4}$. Clearly $\left\langle a^{4}, b^{4}\right\rangle \leq \Phi(X)$. In addition, $|N: \Phi(X)|=|N: X| \mid X$ : $\Phi(X) \mid=2 \cdot 2^{3}=2^{4}$; therefore, we can conclude that $\Phi(X)=\left\langle a^{4}, b^{4}\right\rangle=\Phi(\Phi(N))$. Note that $\left\langle a^{2}, b^{2}\right\rangle$ is a metacyclic maximal subgroup of $X$ and $H_{1}$ is not contained in $\left\langle a^{2}, b^{2}\right\rangle$ since we agreed that $d\left(H_{1}\right)=3$. Therefore, $X=H_{1}\left\langle a^{2}, b^{2}\right\rangle$.

We write $\bar{g}$ to denote the image of $g$ in $\bar{G}=G / \Phi(X)$. Also if $K$ is any subgroup of $G$, then $\bar{K}$ is the image of $K$ in $G$. Applying Lemma 9 to the group $\bar{N}=\langle\bar{a}\rangle\langle\bar{b}\rangle, o(\bar{a})=o(\bar{b})=2^{2}$, we obtain that $\left\langle\bar{a}^{2}, \bar{b}^{2}\right\rangle \leq \mathrm{Z}(\bar{N})$. Since $\bar{X}$ is an elementary abelian group of order 8 contained in $\bar{N}$, it follows that $\bar{N}$ is nonmetacyclic. Hence, $[\bar{a}, \bar{b}]=\bar{a}^{2} \bar{b}^{2}$ by Lemma 9 . Moreover, since $\bar{x}$ centralises $\bar{a}^{2}$ and $\bar{y}$ centralises $\bar{b}^{2}$, we have that $\left\langle\bar{a}^{2}, \bar{b}^{2}\right\rangle \leq \mathrm{Z}(\bar{G})$.

Let us now prove that $\bar{H}$ is nonabelian. Since $y \in G=H N$, there exists $n \in N$ such that $n y \in H$. In addition, there exists $t \in\left\langle a^{2}, b^{2}\right\rangle$ such that $a b t \in H_{1}$.

Bearing in mind that $\bar{n}$ is a product of a power of $\bar{a}$ and a power of $\bar{b}$, we can conclude that $(\bar{a} \bar{b})^{\bar{n}} \in\{\bar{a} \bar{b}, \bar{b} \bar{a}\}$. Therefore,

$$
[\overline{a b t}, \overline{n y}]=[\overline{a b}, \overline{n y}]=(\bar{a} \bar{b})^{-1}(\bar{a} \bar{b})^{\bar{n} \bar{y}}=\bar{b}^{-1} \bar{a}^{-1}(\bar{a} \bar{b})^{\bar{y}} \text { or } \bar{b}^{-1} \bar{a}^{-1}(\bar{b} \bar{a})^{\bar{y}},
$$

which is equal to $\bar{b}^{-2}$ or $[\bar{a}, \bar{b}] \bar{b}^{-2}$. Thus, $[\overline{a b t}, \overline{n y}]=\bar{a}^{2}$ or $\bar{b}^{2}$. Consequently, $[\overline{a b t}, \overline{n y}] \neq 1$ and $\bar{H}$ is nonabelian.

Consequently, $H^{\prime}$ is not contained in $\Phi(X)$. Therefore, $H^{\prime}$ is not contained in $\Phi\left(H_{1}\right)$. In particular, $H / \Phi\left(H_{1}\right)$ is nonabelian and so $\Phi\left(H_{1}\right)<\Phi(H)$. Since $d\left(H_{1}\right)=3$ and $\left|H: H_{1}\right|=2^{2}$, we obtain that $\left|H: \Phi\left(H_{1}\right)\right|=\left|H: H_{1}\right|\left|H_{1}: \Phi\left(H_{1}\right)\right|=2^{5}$. Hence, $|H: \Phi(H)| \leq 2^{4}$ and then $d(H) \leq 4$, contrary to the choice of $H$, which is our final contradiction.

Therefore, $d(H) \leq 4$ for all subgroups $H$ of $G$, and so $r(G) \leq 4$. The proof of the lemma is complete. 
Lemma 11 Let a finite p-group $G=A B$ be the mutually permutable product of the abelian p-subgroups $A$ and $B$. Assume that $\operatorname{Core}_{G}(A)=1$. If $B$ is of ranks, then the rank of $G$ is at most $s+2$ if $p$ is odd and at most $s+3$ if $p=2$.

Proof Since $A \cap B \leq \operatorname{Core}_{G}(A)=1$, it follows that $A$ and $B$ are totally permutable by [4, Proposition 4.1.16]. Let $D=\operatorname{Core}_{G}(B)$. Then $G / D=(A D / D)(B / D)$ is the totally permutable product of the subgroups $A D / D$ and $B / D$ by [4, Lemma 4.1.10]. Clearly, $B / D$ is core-free in $G / D$.

If $p$ is odd, then $A$ and $B / D$ are both cyclic by Lemma 3(i). Applying Lemma 8, we conclude that $r(G / D) \leq 2$. Hence, $r(G) \leq s+2$.

Assume that $G$ is a 2-group. It follows from Lemma 3(ii) that either $A$ is cyclic or $A=$ $\langle a\rangle \times\langle y\rangle$, where $o(y)=2$ and $y$ inverts $B$. If $s \leq 2$, the result follows from Lemma 10 and if $B$ is normal in $G$, then $r(G) \leq s+2$. Therefore, we may assume that $s \geq 3$ and $B / D \neq 1$.

Applying Lemma 3(ii), we have that $d=d(B / D) \leq 2$. Thus, $D \not \leq \Phi(B)$ otherwise $s=d(B / D) \leq 2$. Let $|D: D \cap \Phi(B)|=2^{t}, t \geq 1$. Then

$$
2^{t}=|D \Phi(B): \Phi(B)|=\frac{|B: \Phi(B)|}{|B: D \Phi(B)|} \leq \frac{|B: \Phi(B)|}{|B / D: \Phi(B / D)|}=2^{s-d},
$$

which implies that $t+d \leq s$.

Let $x_{1}(D \cap \Phi(B)), \ldots, x_{t}(D \cap \Phi(B))$ be generators of $D / D \cap \Phi(B)$ and denote $K=$ $\left\langle x_{1}, x_{2}, \ldots, x_{t}\right\rangle \leq D$. Let $x \in K$ and $a \in A$. Then $x^{a} \in\langle a\rangle\langle x\rangle \cap D=\langle x\rangle$. Consequently, $K$ is a normal subgroup of $G$. Since $D=K(D \cap \Phi(B))$, we have that $D / K \leq \Phi(B / K)$ and so $d(B / K)=d(B / D)=d$.

Note that $G / K$ is the totally permutable product of the subgroups $A K / K$ and $B / K$. Furthermore, $r(B / K)=d$, and $A K / K$ is either cyclic or $A K / K=\langle a K\rangle \times\langle y K\rangle$ such that $y K$ inverts $B / K$ and $o(y K)=2$. It follows from Lemma 10 that $r(G / K) \leq r(B / K)+3=$ $d+3$. Hence, $r(G) \leq r(K)+d+3 \leq t+d+3 \leq s+3$.

\section{Proof of main theorems}

Proof of Theorem $A$. Let $H=\left\langle a_{1} b_{1}, \ldots, a_{n} b_{n}\right\rangle$ be a finitely generated subgroup of $G$, where $a_{i} \in A$ and $b_{i} \in B, 1 \leq i \leq n$. Then $H$ is finite as $G$ is locally finite. Moreover, $H$ is soluble since $G$ is metabelian by [4, Theorem 3.1.7]. Let $C=\operatorname{Core}_{G}(A)$. Since $A \cap B \leq C$, it follows that $G / C$ is a totally permutable product of the subgroups $A / C$ and $B C / C$ by $[4$, Lemma 4.1.10 and Proposition 4.1.16],

Write $L=\left\langle a_{1}, \ldots, a_{n}\right\rangle, F=\left\langle b_{1}, \ldots, b_{n}\right\rangle, X=\langle L, F\rangle$ and $D=C \cap X$. Note that all of them are finite and $H \leq X$. Note that $X C / C$ is the totally permutable product of the subgroups $L C / C$ and $F C / C$. Since $X / D$ is isomorphic to $X C / C$, it follows that $X / D$ is a finite group that is the totally permutable product of the subgroups $L D / D$ and $F D / D$ and $L D / D \cap F D / D=1$. Let $p$ be a prime and let $L_{p}$ and $F_{p}$ be the Sylow $p$-subgroups of $L$ and $F$, respectively. Then $U / D=\left(L_{p} D / D\right)\left(F_{p} D / D\right)$ is a Sylow $p$-subgroup of $X / D$. Also, $U / D$ is the totally permutable product of the subgroups $L_{p} D / D$ and $F_{p} D / D$.

Let $S / D=\operatorname{Core}_{U / D}\left(L_{p} D / D\right)$. By [4, Lemma 4.1.10], $U / S$ is the totally permutable product of the subgroups $L_{p} S / S$ and $F_{p} S / S$. Moreover, $L_{p} S / S$ is core-free in $U / S$. By Lemma 11, we get that $r(U / S) \leq r\left(F_{p} S / S\right)+2$ or $r\left(F_{p} S / S\right)+3$ if $p=2$. Since $S \leq L_{p} D \leq$ $A$ and $F_{p} S / S \leq B S / S$, we have that either $r(U) \leq r+s+2$ or $r(U) \leq r+s+3$ if $p=2$. Clearly a Sylow $p$-subgroup $H_{p}$ of $H$ is contained in a conjugate of a Sylow $p$-subgroup of $U$. Therefore, either $d\left(H_{p}\right) \leq r+s+2$ or $d\left(H_{p}\right) \leq r+s+3$ if $p=2$. By Lemma $6, H$ can be generated by $r+s+3$ elements. Consequently, the rank of $G$ is at most $r+s+3$. 
Proof of Theorem B. If $A$ or $B$ is normal in $G, r(G) \leq r+s$. Thus, we may assume that neither $A$ nor $B$ are normal in $G$. Let $C=\operatorname{Core}_{G}(A)$. Since $A \cap B \leq C$, we have that $G / C$ is the totally permutable product of the subgroups $A / C$ and $B C / C$ by [4, Lemma 4.1.10]. Note that $A / C$ is core-free in $G / C$.

Suppose $B C / C$ is weak. Then $B C / C$ is a normal subgroup of $G / C$ with $|G / C: B C / C|=$ 2 by Lemma 4 . Therefore, we have the normal series $1 \leq C \leq B C \leq G$ with the rank of $C$ at most $r=r(A)$, the rank of $B C / C$ at most $s=r(B)$ and $G / B C$ cyclic. Hence, the rank of $G$ is at most $r+s+1$. If $D=\operatorname{Core}_{G}(B)$, a similar argument applies if $A D / D$ is weak.

Thus, we may assume that both $A D / D$ and $B C / C$ are periodic groups. On the other hand, since $A \cap B$ is a normal subgroup of $G$, we have that $A \cap D=A \cap B=C \cap B$. This implies that $A /(A \cap B)$ and $B /(A \cap B)$ are both periodic groups. Denote $Z=A \cap B$. Since $Z$ is abelian of finite rank, we can consider a free abelian subgroup $E$ of $Z$ of maximal rank, $k$ say. Then $Z / E$ is a periodic group of finite rank, and $E / E^{m}$ is a finite group of order $m^{k}$ for every positive integer $m$, where $E^{m}$ is the subgroup generated by $\left\{x^{m}: x \in E\right\}$. Since $E \leq \mathrm{Z}(G)$ we have that $G / E^{m}$ is the product of $A / E^{m}$ and $B / E^{m}$ both periodic groups of finite rank. It then follows that $G / E^{m}$ is locally finite (and of finite rank) by a theorem of Černikov [1, Theorem 3.2.12].

If $E=1$, then $G$ is locally finite. It follows from Theorem A that $r(G) \leq r+s+3$. Assume now that $E \neq 1$. Let $H$ be a finitely generated subgroup of $G$. Since $G / E^{m}$ is locally finite for all positive integers $m$, it follows that $H E^{m} / E^{m} \simeq H /\left(H \cap E^{m}\right)$ is finite and soluble of rank at most $r+s+3$ by Theorem A.

Let $X$ be a subgroup of $H$. Then $X /(X \cap E) \simeq X E / E$ is finite. Moreover, $E$ is finitely generated because it has finite rank. This implies that $X \cap E$ is finitely generated since subgroups of finitely generated abelian groups are finitely generated. Consequently, $X$ is finitely generated and $H$ is polycyclic.

We show next that for every normal subgroup $N$ of $H$ of finite index, there exists a positive integer $m$ such that $H \cap E^{m} \leq N$. Since $H /(N \cap E)$ is finite, we may assume without loss of generality that $N \leq E$. Then $E / N=U / N \times W / N$, where $U / N$ is free abelian and $W / N$ is finite. Assume the exponent of $W / N$ is $m$. Then $(E / N)^{m}=(U / N)^{m}$ is free abelian. Now $\left(H \cap E^{m}\right) N / N \leq H / N$ which is finite. Also $\left(H \cap E^{m}\right) N / N \leq E^{m} N / N \leq(E / N)^{m}$. This implies that $\left(H \cap E^{m}\right) N / N$ is a finite subgroup of a free abelian group. Consequently, $\left(H \cap E^{m}\right) N / N=1$ and $H \cap E^{m} \leq N$, as claimed.

Recall that $H /\left(H \cap E^{m}\right)$ has rank at most $r+s+3$ for all positive integers $m$. It follows that $H / N$ has rank at most $r+s+3$ for every normal subgroup $N$ of $H$ of finite index. Now we can apply Lemma 7 to conclude that $d(H) \leq r+s+4$. Consequently, the rank of $G$ is at most $r+s+4$. The proof of the theorem is now complete.

Acknowledgements The first and third authors are supported by the Grant MTM2014-54707-C3-1-P from the Ministerio de Economía y Competitividad, Spain, and FEDER, European Union. The first and fourth authors are supported by Prometeo/2017/057 of Generalitat, Valencian Community, Spain. The third author is also supported by the predoctoral Grant 201606890006 from the China Scholarship Council. We are grateful to the referee of an earlier version of this paper for comments and suggestions that have lead to improvements in the bounds and their proofs.

\section{References}

1. Amberg, B., Franciosi, S., De Giovanni, F.: Products of Groups, vol. 992. Clarendon Press, Oxford (1992)

2. Amberg, B., Kazarin, L.S.: On the rank of a product of two finite $p$-groups and nilpotent $p$-algebras. Commun. Algebra 27(8), 3895-3907 (1999) 
3. Amberg, B., Sysak, Y.P.: Locally soluble products of two subgroups with finite rank. Commun. Algebra 24(7), 2421-2445 (1996)

4. Ballester-Bolinches, A., Esteban-Romero, R., Asaad, M.: Products of Finite Groups, vol. 53. Walter de Gruyter, Berlin/New York (2010)

5. Baumslag, G., Bieri, R.: Constructable solvable groups. Math. Z. 151(3), 249-257 (1976)

6. Beidleman, J., Heineken, H.: Totally permutable torsion subgroups. J. Group Theory 2, 377-392 (1999)

7. Beidleman, J., Heineken, H.: A survey of mutually and totally permutable products in infinite groups, topics in infinite groups. Quad. Mat 8, 45-62 (2001)

8. Cooper, C.D.: Power automorphisms of a group. Math. Z. 107(5), 335-356 (1968)

9. Dixon, M.R.: Sylow Theory, Formations, and Fitting Classes in Locally Finite Groups, vol. 2. World Scientific, Singapore (1994)

10. Huppert, B.: Endliche Gruppen I, vol. 134. Springer, Berlin, Heidelberg (1967). https://doi.org/10.1007/ 978-3-642-64981-3

11. Janko, Z.: Finite 2-groups with exactly one nonmetacyclic maximal subgroup. Isr. J. Math. 166(1), 313347 (2008)

12. Linnell, P.A., Warhurst, D.: Bounding the number of generators of a polycyclic group. Arch. Math. 37(1), 7-17 (1981)

13. Lucchini, A.: A bound on the number of generators of a finite group. Arch. Math. 53(4), 313-317 (1989)

14. Lucchini, A.: A bound on the presentation rank of a finite group. Bull. Lond. Math. Soc. 29(4), 389-394 (1997) 